\title{
BMJ Open National retrospective cohort study to identify age-specific fatality risks of comorbidities among hospitalised patients with influenza-like illness in Taiwan
}

Ting-Chia Weng, ${ }^{\oplus 1}$ Han-Yi Robert Chiu, ${ }^{2,3}$ Shey-Ying Chen, ${ }^{3,4}$ Fuh-Yuan Shih, ${ }^{3}$ Chwan-Chuen King, ${ }^{4}$ Cheng-Chung Fang ${ }^{\circledR 3}$

To cite: Weng T-C, Chiu H-YR, Chen S-Y, et al. National retrospective cohort study to identify age-specific fatality risks of comorbidities among hospitalised patients with influenza-like illness in Taiwan. BMJ Open 2019;9:e025276. doi:10.1136/ bmjopen-2018-025276

- Prepublication history and additional material for this paper are available online. To view these files, please visit the journal online (http://dx.doi. org/10.1136/bmjopen-2018025276).

T-CW and H-YRC contributed equally.

Received 10 July 2018 Revised 2 May 2019 Accepted 2 May 2019
Check for updates

(C) Author(s) (or their employer(s)) 2019. Re-use permitted under CC BY-NC. No commercial re-use. See rights and permissions. Published by BMJ.

For numbered affiliations see end of article.

Correspondence to Dr Cheng-Chung Fang; conrad@ntu.edu.tw

\section{ABSTRACT}

Objectives This study aimed to examine comprehensively the prognostic impact of underlying comorbidities among hospitalised patients with influenza-like illness (ILI) in different age groups and provide recommendations targeting the vulnerable patients.

Setting and participants A retrospective cohort of 83227 hospitalised cases with ILI were identified from Taiwan's National Health Insurance Research Database from January 2005 to December 2010. Cases were stratified into three different age groups: paediatric (0-17 years), adult (18-64 years) and elderly ( $\geqq 65$ years), and their age, sex, comorbidity and past healthcare utilisation were analysed for ILI-associated fatality.

Main outcome measures ORs for ILI-related fatality in different age groups were performed using multivariable analyses with generalised estimating equation models and adjusted by age, sex and underlying comorbidities.

Results Hospitalised ILI-related fatality significantly increased with comorbidities of cancer with metastasis (adjusted OR (aOR)=3.49, 95\% Cl: 3.16 to 3.86 ), haematological malignancy (aOR=3.02, 95\% Cl: 2.43 to $3.74)$, cancer without metastasis ( $\mathrm{aOR}=1.72,95 \% \mathrm{Cl}$ : 1.54 to 1.91), cerebrovascular ( $\mathrm{aOR}=1.24,95 \% \mathrm{Cl}: 1.15$ to 1.33 ) and heart diseases (aOR=1.19, $95 \% \mathrm{Cl}: 1.11$ to 1.27) for all age groups. Adult patients with AIDS; adult and elderly patients with chronic kidney disease, tuberculosis and diabetes were significantly associated with elevated risk of death. Severe liver diseases and hypothyroidism among elderly, and dementia/epilepsy among elderly and paediatrics were distinctively associated with likelihood of ILI-related fatality.

Conclusions Different age-specific comorbidities were associated with increasing risk of death among hospitalised ILI patients. These findings may help update guidelines for influenza vaccination and other prevention strategies in high-risk groups for minimising worldwide ILI-related deaths.

\section{INTRODUCTION}

The global burden of influenza involves seasonal epidemics and occasional
Strengths and limitations of this study

- The study used a large database, 83227 hospital admissions with influenza-like illness (ILI) from January 2005 to December 2010, providing high statistical power.

- The nationwide cohort design provides high generalisability with comprehensive analysis of 25 clinically important comorbidities covering all the three age groups in paediatrics, adults and elderly.

- Multivariable analyses, performed with generalised estimating equation models and adjusted covariates including age, sex, comorbidities, influenza periods, hospital levels and prior healthcare utilisations, provide independent risk factors of ILI-related death.

- A more complete data on vaccination history, laboratory-confirmed influenza, antiviral treatment, body mass index measurements and smoking status may further improve our models.

pandemics. ${ }^{12}$ As poultry and swine farms have expanded and thus increased dynamic changes in influenza viruses via transmission among different host species, the future threat of novel influenza viruses leading to pandemics may be inevitable. ${ }^{3}$ However, accurately diagnosing influenza virus in suspected patients is a common challenge faced by all clinicians. $^{2}$ Influenza virus infection may not be identified in many instances because influenza virus is only detectable for a short period of time and/or many people do not seek medical care until after the first few days of acute illness. Influenza-like illness (ILI) became a surrogate indicator for practical use in the timely surveillance and measurement of the disease burden of influenza. ${ }^{2}{ }^{4}$ The aim of this study is to assess the prognosis of hospitalised patients with ILI, which includes common presentations of influenza: fever, 
sore throat, headache, musculoskeletal and gastrointestinal symptoms ${ }^{5-7}$ The outcomes from ILI cases are mostly mild or self-limited, but a small percentage of them can develop into severe morbidity or mortality. ${ }^{2}$ The severe cases of ILI typically occur in the very young, the elderly and patients with chronic illness. ${ }^{8-14}$ It is essential to identify, at an early stage, the most vulnerable ILI patients with higher risk to death ${ }^{1516}$ and provide clinical recommendations and public health prevention strategies to minimise fatality.

Elderly and young children, as well as patients with chronic underlying medical conditions, are typically associated with complications of respiratory infections. ${ }^{8} 11 \quad 13 \quad 15 \quad 17$ Clinicians and public health decision-makers are expecting better evidence that requires careful and thorough examining of the roles that comorbidities play in patients of different age groups. The better evidence aims to assist in the prognosis at the beginning of the first medical visit for patients presenting ILI. ${ }^{18-20}$ To date, global guidelines on the prevention and management of ILI-related complications are mostly consensus-based; thus, evidence-based guidelines about the risks of comorbidities have been under investigation. ${ }^{82} 22$

Free influenza vaccination programme in Taiwan started in 1998, for population aged over 65 years. The target vaccination population expanded year after year with the consideration of age, comorbidity and occupational exposure. In 2018, all adults over 50 years and paediatric population from 6 months to 18 years were recommended for vaccination. In addition, high-risk occupations in healthcare, livestock workers, live-poultry market workers and residents in long-term care facilities or prisons and jails were all covered. Furthermore, patients with obesity and chronic underlying diseases and pregnant women are recommended to receive vaccination after physician evaluation. ${ }^{23}$ In this study, using a nationwide cohort data, we aimed to conduct a comprehensive analysis on the age-specific comorbidities associated with fatality on hospitalised patients with ILI.

We believe that the identification of patients with highrisk comorbidities can lead to the support of clinical recommendations and guideline directions for public health policies, including the timely management of antibiotics/antiviral treatments ${ }^{20}$ and other preventions, such as influenza and pneumococcal immunisation ${ }^{321}$ and non-pharmaceutical preventive measures through health education. $^{24} 25$

\section{METHODS}

We conducted a database analysis using the National Health Insurance Research Database (NHIRD) containing records of approximately 1 million patients randomly selected from the 24 million beneficiaries of the National Health Insurance in Taiwan. The database collects encrypted claim records of emergency department (ED) patient, outpatient and inpatient data for billing purposes. The nationwide cohort study was conducted using the diagnostic codes of the International Classification of Diseases, Ninth Revision, Clinical Modification (ICD-9-CM).$^{26}$ Hospitalised ILI patients from January 2005 to December 2010 were included for data analyses. The definition of ILI was based on Taiwan Emergency Department-Based Syndromic Surveillance System. ${ }^{4}$ We added six codes (382.9, 461.9, 465.8, 466.0, 487.1, 780.6) after comparing the code-based syndromic surveillance for ILI from the USA. ${ }^{27}$ The codes are presented in online supplementary table 1 . In the instances where the patient had been re-admitted to the hospital with ILI within 14 days from a previous discharge of ILI-related hospitalisation, the two admissions were combined as the same episode. The flow diagram of the selection process of the study subjects is shown in figure 1 .

Comorbidities included in the study analyses were selected after a literature review and a group discussion among physicians specialising in infection and emergency medicine, paediatrics, occupational medicine, and public health professionals. The comorbidities related to malignant diseases were defined, based on the records from the Taiwan Catastrophic Illness Card (TCIC) codes for malignant diseases both 12 months before and 6 months after the first ILI admission date. We included pregnant women when the first date of hospitalisation with ILI occurred up to 3 months before delivery/abortion. We included postpartum women when delivery/ abortion occurred up to 3 months before the first date of ILI hospitalisation. Data on other type of comorbidities were collected through diagnoses from reimbursement records 12 months before the first date of ILI admission. A comorbidity was confirmed with at least one diagnosis of hospitalisation claim or through two or more diagnoses from outpatient and/or ED visits. Grouping of comorbidity with ICD-9-CM codes was modified from the Charlson $e t a l,{ }^{28}$ Deyo $e t a l^{29}$ and Elixhauser $e t a l^{30}$ measurements using the most frequently applied codes. For autoimmune disease, organ transplantation, congenital immunodeficiency and malignant, hepatic and mental diseases, we also confirmed the disease status through the TCIC information to improve specificity. Definition of each comorbidity is shown in online supplementary table 2. The ILI-associated fatalities were the main outcome of interest. Fatality case was defined by discharge information recorded in the NHIRD as 'hospital mortality' (discharge transfer code ' 4 '). Additionally, we also included cases that discharged under critical conditions (discharge transfer code 'A'), who also withdrew from the National Health Insurance and had not received any healthcare services in the following 12 months after hospital discharge. All analyses were performed in the overall study population, as well as stratified by three age groups: paediatric patients (0-17 years), adult patients (18-64 years) and elderly patients ( $\geqq 65$ years).

In total, 25 clinically important comorbidities were selected after panel discussion and logistic regression analyses were used to evaluate the significance of individual comorbidity. Furthermore, the outcome measures 
Hospitalised cases with one or more ILI-related ICD-9-CM code(s)

from 2005 to 2010 in the NHIRD $(\mathrm{N}=92,376)$

\section{Inclusion Criteria:}

1. Patients with comorbidity, pregnancy or postpartum.

2. Linked with registry of "catastrophic illness" to include patients with autoimmune disease, organ transplantation, congenital immunodeficiency, and malignant, hepatic, and mental diseases.

Patients with ILI-related hospitalisation in 2005-2010 (N=90,325)

\section{Exclusion:}

Missing data for hospital accreditation

level $(\mathrm{N}=882)$

Patients with ILI-related hospitalisation in 2005-2010 with available information of all the study variables $(\mathrm{N}=89,439)$.

The interval of the indexed two ILI admissions occurring within 14 days were regarded as a single ILI episode $(\mathrm{N}=6,212)$.

LI-related hospitalisation episodes in 2005-2010 ( $\mathrm{N}=83,227)$.

Figure 1 Flow diagram of the selection process of the study population. ICD-9-CM, International Classification of Diseases, Ninth Revision, Clinical Modification; ILI, influenza-like illness; NHIRD, National Health Insurance Research Database in Taiwan.

were stratified by influenza season months (December to April) and non-influenza season months ${ }^{31}$ to compare and evaluate the fatalities by different comorbidities in hospitalised ILI patients. Finally, we performed multivariable analyses to measure the independent predictability of death from these comorbidities among the three age groups. We used stepwise logistic regression analyses with forward selection method at the entry criteria of $\mathrm{p}<0.15$ to achieve a good model fit. ${ }^{32}{ }^{33}$ Stepwise logistic regression analyses selected 16 comorbidities in the 'all patients group', 9 in the 'paediatric patient group', 13 in the 'adult patient group' and 17 in the 'elderly patient group'. We performed generalised estimating equation
(GEE) models to adjust for the hospital clustering effect and repeated hospitalisations as patients had two or more ILI-related admissions within an ILI episode. ${ }^{32}$ The variables adjusted in the GEE model included age, sex and different study periods (from previous year's July to next year's June), patient-admitted hospital levels (local hospital, regional hospital or medical centre), and prior frequencies of hospitalisation in the past 12 months $(0,1-2$ or $>3$ admissions). The statistical analyses were performed using SAS V.9.4 (SAS Institute, Cary, North Carolina, USA). A two-tailed $\mathrm{p}<0.05$ was considered statistically significant. 
Table 1 Demographic data and proportions of comorbidities in patients hospitalised with influenza-like illness among the three age groups and all patients in Taiwan, 1 January 2005 to 31 December 2010

\begin{tabular}{|c|c|c|c|c|c|}
\hline Age groups (years) & All patients & Paediatric (0-17) & Adult (18-64) & Elderly ( $\geq 65)$ & \\
\hline Population, $\mathbf{N}$ ( $\%$ of all) & $83227(100.00)$ & $19847(23.85)$ & 25988 (31.23) & 37392 (44.93) & \\
\hline $\mathbf{N}(\%)$ of variable & $\mathbf{N}(\%)$ & $\mathbf{N}(\%)$ & $\mathrm{N}(\%)$ & $\mathrm{N}(\%)$ & P value* \\
\hline \multicolumn{6}{|l|}{ Gender } \\
\hline Female & $33488(40.24)$ & $8739(44.03)$ & $11263(43.34)$ & $13486(36.07)$ & $<0.001$ \\
\hline Male & $49739(59.76)$ & $11108(55.97)$ & $14725(56.66)$ & 23906 (63.93) & \\
\hline Deaths & $5282(6.35)$ & $37(0.19)$ & $984(3.79)$ & $4261(11.40)$ & $<0.001$ \\
\hline \multicolumn{6}{|l|}{ Comorbidities } \\
\hline Heart disease & $21776(26.16)$ & $101(0.51)$ & $3945(15.18)$ & $17730(47.42)$ & $<0.001$ \\
\hline PVD & $1689(2.03)$ & $5(0.03)$ & $292(1.12)$ & $1392(3.72)$ & $<0.001$ \\
\hline Hypertension & $30663(36.84)$ & $28(0.14)$ & $6726(25.88)$ & 23909 (63.94) & $<0.001$ \\
\hline Hyperlipidaemia & $7171(8.62)$ & $25(0.13)$ & $2924(11.25)$ & $4222(11.29)$ & $<0.001$ \\
\hline CVA & 16935 (20.35) & $33(0.17)$ & $2843(10.94)$ & $14059(37.60)$ & $<0.001$ \\
\hline Dementia/Epilepsy & $8443(10.14)$ & $471(2.37)$ & $1214(4.67)$ & $6758(18.07)$ & $<0.001$ \\
\hline COPD/Asthma & 32646 (39.23) & $4564(23.00)$ & $5830(22.43)$ & $22252(59.51)$ & $<0.001$ \\
\hline Allergic rhinitis & $10713(12.87)$ & $4894(24.66)$ & $3307(12.73)$ & $2512(6.72)$ & $<0.001$ \\
\hline Autoimmune disease & $635(0.76)$ & $29(0.15)$ & $336(1.29)$ & $270(0.72)$ & $<0.001$ \\
\hline Severe liver disease & $289(0.35)$ & $0(0.00)$ & $206(0.79)$ & $83(0.22)$ & $<0.001$ \\
\hline Peptic ulcer disease & $14514(17.44)$ & $119(0.60)$ & $4150(15.97)$ & $10245(27.40)$ & $<0.001$ \\
\hline Diabetes & $16416(19.72)$ & $35(0.18)$ & $4298(16.54)$ & $12083(32.31)$ & $<0.001$ \\
\hline Gout & $4673(5.61)$ & $12(0.06)$ & $1385(5.33)$ & $3276(8.76)$ & $<0.001$ \\
\hline Hyperthyroidism & $542(0.65)$ & $5(0.03)$ & $297(1.14)$ & $240(0.64)$ & $<0.001$ \\
\hline Hypothyroidism & $558(0.67)$ & $31(0.16)$ & $174(0.67)$ & $353(0.94)$ & $<0.001$ \\
\hline CKD & $6617(7.95)$ & $70(0.35)$ & $1400(5.39)$ & $5147(13.76)$ & $<0.001$ \\
\hline Cancer without metastasis & $4113(4.94)$ & $60(0.30)$ & $1217(4.68)$ & $2836(7.58)$ & $<0.001$ \\
\hline Metastatic cancer & $4264(5.12)$ & $34(0.17)$ & $1863(7.17)$ & $2367(6.33)$ & $<0.001$ \\
\hline Haematological malignancy & $922(1.11)$ & $147(0.74)$ & $333(1.28)$ & $442(1.18)$ & $<0.001$ \\
\hline Congenital immunodeficiency & $30(0.04)$ & $23(0.12)$ & $7(0.03)$ & $0(0.00)$ & $<0.001$ \\
\hline Organ transplantation & $123(0.15)$ & $4(0.02)$ & $100(0.38)$ & $19(0.05)$ & $<0.001$ \\
\hline AIDS & $63(0.08)$ & $3(0.02)$ & $57(0.22)$ & $3(0.01)$ & $<0.001$ \\
\hline Tuberculosis & $3083(3.70)$ & $34(0.17)$ & $713(2.74)$ & $2336(6.25)$ & $<0.001$ \\
\hline Mental illness & $1821(2.19)$ & $39(0.20)$ & $1211(4.66)$ & $571(1.53)$ & $<0.001$ \\
\hline Pregnancy/Post partum & $334(0.40)$ & $3(0.02)$ & $331(1.27)$ & $0(0.00)$ & $<0.001$ \\
\hline
\end{tabular}

${ }^{*} \mathrm{X}^{2}$ test to evaluate whether there would be a significant difference among the three age groups.

CKD, chronic kidney disease; COPD, chronic obstructive pulmonary disease; CVA, cerebrovascular accident; PVD, peripheral vascular disease.

Patient and public involvement statement

This study was conducted by using a National Health Insurance claims database. All records were de-identified and there were no patients' identifiable information.

\section{RESULTS}

In total, 83227 events of ILI-related hospitalisation from 1 January 2005 to 31 December 2010 were included for analysis. The demographic data are shown in table 1 .
There were significantly more male than female ILI cases with a ratio of $1.49(\mathrm{p}<0.001)$. The majority of ILI-related admissions were elderly patients (44.93\%), followed by adult patients $(31.23 \%)$, then paediatric patients $(23.85 \%)$. We identified 5282 deaths $(6.35 \%)$ in our study population, where the case fatality rate of these hospitalised ILI cases was significantly higher among elderly patients $(11.40 \%)$ than among adult $(3.79 \%)$ and paediatric patients $(0.19 \%)(\mathrm{p}<0.001)$. Among the overall comorbidities, chronic obstructive pulmonary disease 
(COPD) /asthma (39.23\%) was the top prevalent comorbidity, followed by hypertension $(36.84 \%)$, heart disease (26.16\%), cerebrovascular accident (CVA) $(20.35 \%)$ and diabetes $(19.72 \%)$.

Logistic regression analyses were used to evaluate the significance of the 25 comorbidities relating to ILI-associated fatality in patients of different age groups. The case fatality rate and univariate OR of each comorbidity are summarised in table 2. Ten comorbidities showed small difference in proportions between influenza and non-influenza seasons among hospitalised ILI cases with statistical differences in table 3. Among them, three comorbidities (cardiovascular diseases, dementia/ epilepsy and tuberculosis) demonstrated slightly higher case fatality rates during influenza seasons than those in non-influenza seasons with statistical significance.

The results of the multivariable analysis by GEE models are shown in figure 2. In all patients, the highest adjusted OR (aOR) of ILI-associated fatality was in those patients with AIDS (aOR 6.53, 95\% CI: 2.89 to 14.76), followed by those with metastatic cancer (aOR 3.49, 95\% CI: 3.16 to 3.86), haematological malignancy (aOR $3.02,95 \% \mathrm{CI}$ : 2.43 to 3.74 ) and solid organ cancer without metastasis (aOR 1.72, 95\% CI: 1.54 to 1.91 ). Other high-risk underlying medical conditions with statistical significance included chronic kidney disease (CKD) (aOR 1.57, 95\% CI: 1.44 to 1.71 ), tuberculosis (aOR $1.37,95 \% \mathrm{CI}$ : 1.22 to 1.54 ), hypothyroidism (aOR 1.37, 95\% CI: 1.04 to 1.79 ), CVA (aOR 1.24, 95\% CI: 1.15 to 1.33 ), peripheral vascular disease (PVD) (aOR 1.22, 95\% CI: 1.05 to 1.42), diabetes (aOR 1.22, 95\% CI: 1.13 to 1.30 ), heart diseases (aOR $1.19,95 \%$ CI: 1.11 to 1.27 ) and dementia/epilepsy (aOR 1.12, 95\% CI: 1.03 to 1.22). However, hypertension (aOR $0.75,95 \%$ CI: 0.70 to 0.81 ), hyperlipidaemia (aOR $0.69,95 \%$ CI: 0.62 to 0.76 ) and allergic rhinitis (aOR 0.61 , $95 \%$ CI: 0.54 to 0.70 ) showed decreased risk of ILI-related deaths.

For the risk of age-specific comorbidity, we further stratified our study population into three age groups. In paediatric patients, hypertension (aOR 23.82, 95\% CI: 6.84 to 82.97 ) and dementia/epilepsy (aOR 4.87, 95\% CI: 1.83 to 13.01 ) were distinctively associated with increased risk of ILI-associated deaths $(\mathrm{p}<0.05)$ (figure 2). In adult patients, AIDS (aOR 9.34, 95\% CI: 4.18 to 20.91), CKD (aOR $1.85,95 \%$ CI: 1.44 to 2.36 ), tuberculosis (aOR 1.89 , 95\% CI: 1.41 to 2.55 ) and diabetes (aOR 1.50, 95\% CI: 1.26 to 1.80) displayed significantly increased risk of ILI-associated deaths $(\mathrm{p}<0.001)$, whereas hyperlipidaemia showed decreased risk (figure 2). In elderly patients, hypothyroidism (aOR 1.48, 95\% CI: 1.11 to 1.98 ) and severe liver disease (aOR 1.94, 95\% CI: 1.13 to 3.30) showed significantly increased risk of ILI-associated deaths $(\mathrm{p}<0.05)$ (figure 2).

\section{DISCUSSION}

This study, using a large national cohort dataset with simultaneously adjusted clinically important comorbidities and several epidemiological covariates, is the first to demonstrate the independent effect of certain age-specific comorbidities on fatality risk among hospitalised ILI patients. Identifying high-risk patients with ILI or influenza to support evidence-based preventive strategies has been advocated in many high-income countries. $^{8}{ }^{13}$ Although meta-analyses have highlighted the important effect of comorbidities on influenza, most of these observational studies have either lacked power or inadequately adjusted for important covariates and thus provided limited evidence. ${ }^{811} 13$ Moreover, most of the published guidelines regarding ILI preventive strategies have targeted elderly populations or those over 50 years of age, thus limiting the applicability of these guidelines to other age groups. ${ }^{34-36}$ Furthermore, previous studies found that several comorbidities were associated with the increased fatality of ILI patients ${ }^{813}$; however, their analyses did not fully control for important covariates (e.g., age, sex and relevant comorbidities). Our results showed that the contribution of a comorbidity to fatality risk among hospitalised ILI patients was age-specific.

Host defence responses, targeting viral entry, transcription, translation, replication and extracellular release, can limit the disease severity and spread of influenza virus infection. ${ }^{37}$ We found that malignant diseases were associated with increased fatality rate among hospitalised ILI patients across all three age groups. The trends of aORs were also consistent in all three groups, with the highest trend being cancer with metastasis, followed by haematological malignancy and cancer without metastasis. Because cancer patients tend to have more depressed immunity directly from malignancy or indirectly from anticancer therapy-related immunodeficiency, our study again highlights the important role of malignant diseases, particularly cancer with metastasis, in contributing to increased fatality risk among hospitalised patients with ILI. Furthermore, heart disease and CVA were another two risky comorbidities across the three age groups. Patients with cardiovascular diseases were associated with increased risk of mortality during influenza epidemics, possibly due to the exacerbation of limited cardiovascular reserve from silent or fulminant viral myocarditis or the induction of acute myocardial infarction in patients with pre-existing coronary artery disease. ${ }^{38}$ The three comorbidities of cancer, heart disease and CVA with higher prevalence rates in all age groups provide evidence-based recommendations for clinical management as well as public health planning.

However, age-specific differences were present in several comorbidities associated with ILI-related fatality. AIDS was an independent predictor for death only in hospitalised adult ILI patients. Other chronic diseases, such as tuberculosis, diabetes and CKD, predicted hospital fatality in both adult and elderly patients and showed similar trends in increasing ILI-associated fatality. In fact, ILI patients with co-infection of HIV are associated with a higher risk of superimposed bacterial infections and increased severity of influenza. ${ }^{40-42}$ Patients with tuberculosis have 


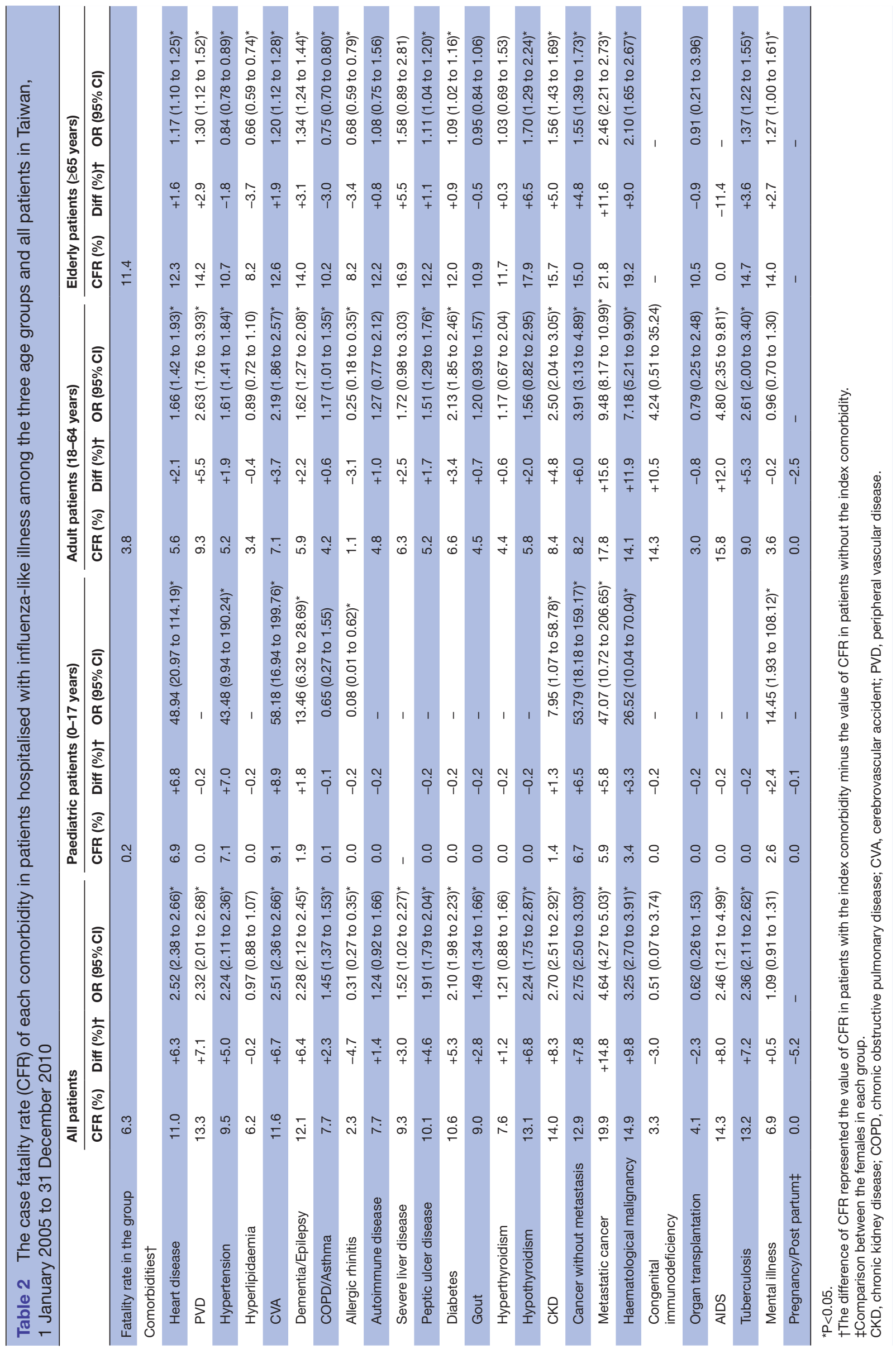


Table 3 Proportions of comorbidity and case fatality rates (CFRs) of comorbidity in patients hospitalised due to influenza-like illness in Taiwan, 1 January 2005 to 31 December 2010

\begin{tabular}{|c|c|c|c|c|c|c|}
\hline \multirow{3}{*}{$\begin{array}{l}\text { Population, } \mathrm{N} \text { (\% of } \\
\text { all) }\end{array}$} & \multicolumn{3}{|c|}{ Proportion of comorbidity } & \multicolumn{3}{|c|}{ CFR of comorbidity } \\
\hline & Influenza season† & $\begin{array}{l}\text { Non-influenza } \\
\text { season }\end{array}$ & & $\begin{array}{l}\text { Influenza } \\
\text { seasont }\end{array}$ & $\begin{array}{l}\text { Non-influenza } \\
\text { season }\end{array}$ & \\
\hline & $36197(43.49)$ & $47030(56.51)$ & & 36197 (43.49) & $47030(56.51)$ & \\
\hline Comorbidity & $\mathbf{N}(\%)$ & $\mathbf{N}(\%)$ & $P$ value & $\begin{array}{l}\text { Fatality (N) } \\
\text { CFR (\%) }\end{array}$ & $\begin{array}{l}\text { Fatality (N) } \\
\text { CFR (\%) }\end{array}$ & P value \\
\hline Heart disease & $9339(25.80)$ & $12437(26.44)$ & $0.036^{*}$ & 1064 (11.39) & 1336 (10.74) & 0.129 \\
\hline PVD & $716(1.98)$ & $973(2.07)$ & 0.357 & $95(13.27)$ & $130(13.36)$ & 0.956 \\
\hline Hypertension & 12897 (35.63) & 17766 (37.78) & $<0.0001^{*}$ & $1246(9.66)$ & $1673(9.42)$ & 0.472 \\
\hline Hyperlipidaemia & $2988(8.25)$ & $4183(8.89)$ & $0.001^{*}$ & $197(6.59)$ & $247(5.90)$ & 0.233 \\
\hline CVA & $6996(19.33)$ & 9939 (21.13) & $<0.0001^{*}$ & 869 (12.42) & $1102(11.09)$ & $0.008^{*}$ \\
\hline Dementia/Epilepsy & 3481 (9.62) & $4962(10.55)$ & $<0.0001^{*}$ & 452 (12.98) & $571(11.51)$ & $0.041^{*}$ \\
\hline COPD/Asthma & $14198(39.22)$ & 18448 (39.23) & 0.996 & $1124(7.92)$ & $1397(7.57)$ & 0.248 \\
\hline Allergic rhinitis & $4686(12.95)$ & 6027 (12.82) & 0.577 & $110(2.35)$ & $133(2.21)$ & 0.628 \\
\hline Autoimmune disease & $275(0.76)$ & $360(0.77)$ & 0.925 & $18(6.55)$ & $31(8.61)$ & 0.334 \\
\hline Severe liver disease & $123(0.34)$ & $166(0.35)$ & 0.749 & $14(11.38)$ & $13(7.83)$ & 0.305 \\
\hline Peptic ulcer disease & $6153(17.00)$ & $8361(17.78)$ & $0.003^{*}$ & $645(10.48)$ & $821(9.82)$ & 0.190 \\
\hline Diabetes & $6930(19.15)$ & $9486(20.17)$ & $<0.0001^{*}$ & $756(10.91)$ & $977(10.30)$ & 0.209 \\
\hline Gout & $1980(5.47)$ & $2693(5.73)$ & 0.112 & $194(9.80)$ & $225(8.35)$ & 0.088 \\
\hline Hyperthyroidism & $230(0.64)$ & $312(0.66)$ & 0.619 & $16(6.96)$ & $25(8.01)$ & 0.646 \\
\hline Hypothyroidism & $225(0.62)$ & $333(0.71)$ & 0.130 & $32(14.22)$ & $41(12.31)$ & 0.512 \\
\hline CKD & $2829(7.82)$ & $3788(8.05)$ & 0.207 & 419 (14.81) & $508(13.41)$ & 0.105 \\
\hline $\begin{array}{l}\text { Cancer without } \\
\text { metastasis }\end{array}$ & $1640(4.53)$ & $2473(5.26)$ & $<0.0001^{*}$ & $204(12.44)$ & $325(13.14)$ & 0.510 \\
\hline Metastatic cancer & $1722(4.76)$ & $2542(5.41)$ & $<0.0001^{*}$ & $347(20.15)$ & 503 (19.79) & 0.771 \\
\hline $\begin{array}{l}\text { Haematological } \\
\text { malignancy }\end{array}$ & $372(1.03)$ & $550(1.17)$ & 0.053 & $58(15.59)$ & $79(14.36)$ & 0.607 \\
\hline $\begin{array}{l}\text { Congenital } \\
\text { immunodeficiency }\end{array}$ & $13(0.04)$ & $17(0.04)$ & 0.986 & $1(7.69)$ & $0(0.00)$ & 0.245 \\
\hline Organ transplantation & $58(0.16)$ & $65(0.14)$ & 0.412 & $3(5.17)$ & $2(3.08)$ & 0.557 \\
\hline AIDS & $26(0.07)$ & $37(0.08)$ & 0.722 & $3(11.54)$ & $6(16.22)$ & 0.601 \\
\hline Tuberculosis & $1264(3.49)$ & $1819(3.87)$ & $0.004^{*}$ & $188(14.87)$ & 220 (12.09) & $0.025^{*}$ \\
\hline Mental illness & $774(2.14)$ & $1047(2.23)$ & 0.390 & $57(7.36)$ & $68(6.49)$ & 0.468 \\
\hline $\begin{array}{l}\text { Pregnancy/ } \\
\text { Post partum }\end{array}$ & $150(0.41)$ & $184(0.39)$ & 0.600 & $0(0.00)$ & $0(0.00)$ & - \\
\hline
\end{tabular}

${ }^{*} \mathrm{P}<0.05$.

†Influenza season was defined as the patients hospitalised on index date from 1 December to 30 April of next year; non-influenza season was defined as the patients admitted on the date other than influenza season months.

$\ddagger \mathrm{X}^{2}$ test between influenza seasons and non-influenza seasons.

also proven to be associated with increased influenza deaths in both the 1918 pandemic $^{43}$ and seasonal influenza. ${ }^{44}$ Lost immune homeostasis of patients with CKD during influenza infection may result in further renal cell damage, ${ }^{45}$ eventually leading to kidney failure and subsequent fatality. In hospitalised ILI adult patients, metabolic diseases have more impact on deaths. A previous study found that diabetes tripled the risk of hospitalisation and quadrupled the risk of intensive care unit admission during the 2009 pandemic influenza. ${ }^{46}$ All these data illustrate the importance of considering preventive measures for age-specific immunocompromised patients to prevent subsequent ILI fatality.

Infants and young children with pre-existing conditions, particularly neurological disorders, are over-represented among influenza-associated paediatric deaths. ${ }^{47} 48$ However, previous studies were mostly conducted with univariable analysis without using multivariable analysis 


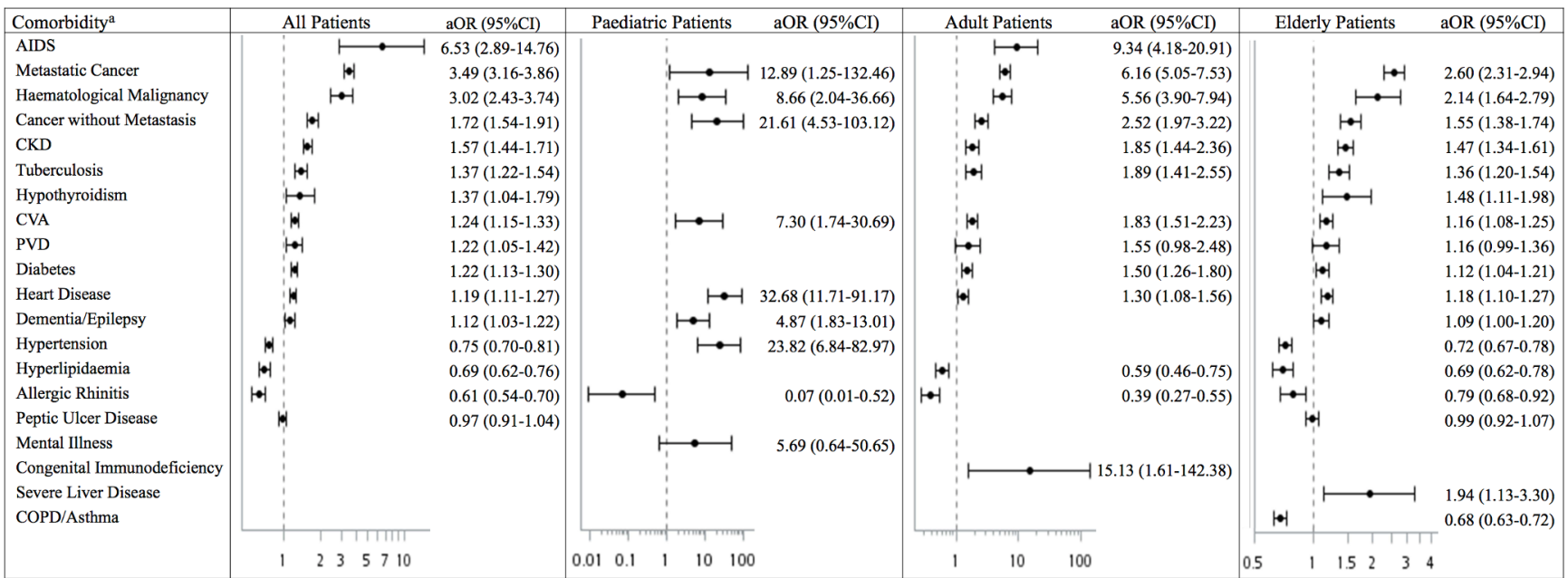

Figure 2 Adjusted OR (aOR) of death in admitted patients with influenza-like illness among the three age groups and all patients in Taiwan, 1 January 2005 to 31 December 2010. CKD, chronic kidney disease; COPD, chronic obstructive pulmonary disease; CVA, cerebrovascular accident; PVD, peripheral vascular disease.

to adjust for other comorbidities; hence the level of evidence was low. ${ }^{811}$ Our study showed that epilepsy was a specific risk for paediatric patients. However, epilepsy was not documented as a risk in a previous meta-analysis. ${ }^{8}$ In addition, hypertension was another significant comorbidity risk only in paediatric ILI cases. The identification of 2 deaths among the 28 paediatric hypertension cases led to a high fatality rate. However, these two fatal cases also showed other comorbidities-one had leukaemia, while the other had moyamoya disease. Certainly, there was not enough evidence to derive any conclusions on hypertension in the paediatric group. Conversely, epilepsy/dementia and hypothyroidism were the specific high-risk comorbidities for elders in this study. A previous meta-analysis showed increased risk of epilepsy/dementia in seasonal and pandemic influenza patients and also indicated that endocrinological disease documented increased risk of death in seasonal influenza patients. ${ }^{8}$ However, this meta-analysis did not specify which endocrinological disease. By contrast, we addressed hypothyroidism to be the most probable disease predisposing fatality among the endocrinological diseases.

On the other hand, several comorbidities were associated with a reduced case fatality rate of ILI either for all age groups (allergic rhinitis) or in a specific age group (e.g., COPD/asthma in the elderly group). ILI patients with allergic rhinitis and COPD/asthma may be more likely to be hospitalised when they presented more prominent respiratory manifestations. ${ }^{49}$ Conversely, the non-infectious airway symptoms caused by allergic rhinitis and asthma/ COPD could also mimic ILI, resulting in increased ILI-related hospitalisation. Our study showed a lower mortality risk for COPD/asthma in the study population of hospitalised ILI patients in Taiwan. We should carefully interpret the results since that risk was different from outpatient setting. The hospitalised patients in our study was $39.23 \%$ with COPD/asthma (table 1). However, the prevalence of COPD was about $6 \%,{ }^{51}$ and the prevalence of asthma was about $11 \%^{52}$ among general population in Taiwan. Certainly, it is important to consider probable mitigation efforts, including vaccination and antiviral medication to alleviate disease burden. ${ }^{23}{ }^{53}$ Adult and elderly ILI patients with hyperlipidaemia associated with lower case fatality rates could be explained by the use of statin to treat hyperlipidaemia. Pre-admission statin use has been shown to reduce 30-day mortality of less severe sepsis patients. ${ }^{54}$ Therefore, the use of statin in hyperlipidaemia patients may benefit the outcome of ILI. As hypertension is a common comorbidity in adult and elderly populations, our results adjusted for covariates and other comorbidities, thereby demonstrating that hypertension had no influence on adult ILI case fatality and decreased elderly ILI-related fatality. These findings are different from those of a previous meta-analysis showing that hypertension increased all-cause mortality in pandemic influenza patients. ${ }^{8}$ Certainly, adjusting for other comorbidities should be important in generating final conclusions for patients with multiple comorbidities.

This study had several limitations. First, our study population involved ILI cases (rather than laboratory-confirmed influenza cases) that have more variations in terms of virulence, vaccination and antibacterial/antifungal/antiviral treatment to influenza and other respiratory pathogens. The fatality risk assessment of ILI in our study was not equal to influenza. It is difficult to obtain laboratory confirmation for every case of influenza in clinical settings due to virological identifications for influenza are time-consuming, expensive and impractical to cover all suspected cases with mild outcomes. Therefore, studying patients with ILI provided more clinically relevant scenarios for physicians to identify vulnerable patients. Second, the severity of comorbidity, compliance of medical treatment and possible mitigation efforts of vaccination, early admission and antiviral treatment could not be evaluated in this study. The coding-associated diagnostic error ${ }^{55}$ may also have limited the accuracy of our disease/comorbidity diagnosis. Third, the comorbidities that we selected here according to the literature review and 
subsequent group discussion may still have had limitations, such as being unstudied or lower prevalent comorbidities. Obesity is associated with increased risk of mortality following influenza infections. ${ }^{56-58}$ However, obesity, immunisation and tobacco use were seldom coded in the National Health Insurance system. Fourth, the aetiology of ILI with/without co-infections of other microbial agents and environmental factors may also have influenced the ILI prognoses..$^{59-61}$ Lastly, infants aged under 1 year have distinctive physiology and immunology responses to infections. The prognosis in this age group may be different from other young children, but subgroup analysis could not be done for limited cases.

The prevalence of comorbidities and environmental factors varied across the globe, with variations on national guidelines. ${ }^{22} 34-3662$ The current vaccination guideline in Taiwan suggested patients with chronic underlying diseases, obesity and pregnant women to be evaluated for influenza vaccination, but the level of evidence for high-risk comorbidities was low. ${ }^{8}$ Our study analysed a national dataset in Taiwan with good representativeness and well-adjusted covariates. The results could support a reference of vulnerable groups to target, which may reduce the burden and prevent the ILI fatalities caused by influenza and other respiratory infectious disease agents. ${ }^{63}{ }^{64}$ Since early antiviral treatment may be beneficial for patients with severe influenza, ${ }^{65-67}$ our findings may assist policy makers in amending guidelines to promptly prescribe antiviral medications for high-risk individuals during the influenza season or pandemic outbreak. Therefore, we offer the following recommendations: first, higher priority should be given to patients with malignancy, heart disease, CVA and AIDS. Second, adult and elderly patients with tuberculosis, diabetes and CKD should also receive more attention. Third, paediatric patients with epilepsy and elderly patients with dementia and hypothyroidism should also be treated with more attention.

In conclusion, our study confirmed that age-specific chronic medical conditions were associated with increased fatalities in hospitalised ILI patients. In the era of personalised medicine and an increasingly ageing population involving various comorbidities, we suggest future studies to calculate the combined risks of comorbidities in an individual patient for better prediction and case management.

\section{Author affiliations \\ ${ }^{1}$ Department of Occupational and Environmental Medicine, National Cheng Kung University Hospital, College of Medicine, National Cheng Kung University, Tainan, Taiwan \\ ${ }^{2}$ Technical Mission of Republic of China (Taiwan) to the Republic of the Marshall Islands, International Cooperation and Development Fund, Taipei, Taiwan ${ }^{3}$ Department of Emergency Medicine, National Taiwan University Hospital and College of Medicine, National Taiwan University, Taipei, Taiwan \\ ${ }^{4}$ Institute of Epidemiology and Preventive Medicine, College of Public Health, National Taiwan University, Taipei, Taiwan}

Acknowledgements The authors would like to thank Dr Ping-Ing Lee at the Department of Pediatrics, National Taiwan University Hospital for his professional guidance in the pediatric data discussion, and Dr Jui-Hsiang Lin at the Institute of Epidemiology and Preventive Medicine, National Taiwan University for double checking the statistical sections. The authors would like to express their sincere gratitude to the Multinational Influenza Seasonal Mortality Study (MISMS) for holding the 2015 and 2016 Influenza Workshops in Taipei and Bethesda, respectively, which were sponsored by the Fogarty International Center of the US National Institutes of Health (NIH). The authors would like to thank Drs Cecile Viboud, Andrew Rambaut, Gerardo Chowell and Winda Liviya $\mathrm{Ng}$ for their very useful exchanges, research stimulation and discussion. In addition, past influenza research experiences learned from annual meetings of Excellence for Influenza Research and Surveillance (CEIRS) were also very useful in considering the future needs for global health. The administrative assistance of Mr. Min-Shiao Hu, and English editing by the Uni-edit (www.uni-edit.net) and Dr Elias F Onyoh are highly appreciated.

Contributors Conceived and designed this study: C-CF, C-CK, F-YS, S-YC, H-YRC and T-CW. Analysed the data: H-YRC. Wrote this manuscript: T-CW, H-YRC, S-YC, F-YS, C-CK and C-CF. Initiated this study: C-CK and C-CF. Revised this manuscript: S-YC, F-YS, C-CK and C-CF. All authors critically revised the manuscript and agreed to the final version.

Funding Travelling to participate at influenza-related meetings was partially supported by the Taiwan's Ministry of Science and Technology (Grant \#103-2621M-002-004, 105-2321-B-002 -025), the Fogarty International Center and National Cheng Kung University Hospital.

\section{Competing interests None declared.}

Patient consent for publication Not required.

Ethics approval The study protocol was approved by the Research Ethics Committee of the National Taiwan University Hospital (ID: 201603086RINB, 14 April 2016).

Provenance and peer review Not commissioned; externally peer reviewed.

Data sharing statement All the de-identified data could be obtained from the National Health Insurance Administration, Ministry of Health and Welfare in Taiwan, Republic of China.

Open access This is an open access article distributed in accordance with the Creative Commons Attribution Non Commercial (CC BY-NC 4.0) license, which permits others to distribute, remix, adapt, build upon this work non-commercially, and license their derivative works on different terms, provided the original work is properly cited, appropriate credit is given, any changes made indicated, and the use is non-commercial. See: http://creativecommons.org/licenses/by-nc/4.0/.

\section{REFERENCES}

1. Cox NJ, Subbarao K. Global epidemiology of influenza: past and present. Annu Rev Med 2000;51:407-21.

2. Hayward AC, Fragaszy EB, Bermingham A, et al. Comparative community burden and severity of seasonal and pandemic influenza: results of the Flu Watch cohort study. Lancet Respir Med 2014;2:445-54.

3. Fineberg HV. Pandemic preparedness and response-lessons from the H1N1 influenza of 2009. N Engl J Med 2014;370:1335-42.

4. Marsden-Haug N, Foster VB, Gould PL, et al. Code-based syndromic surveillance for influenzalike illness by International Classification of Diseases, Ninth Revision. Emerg Infect Dis 2007;13:207-16.

5. Boivin G, Hardy I, Tellier G, et al. Predicting influenza infections during epidemics with use of a clinical case definition. Clin Infect Dis 2000;31:1166-9.

6. Shah SC, Rumoro DP, Hallock MM, et al. Clinical predictors for laboratory-confirmed influenza infections: exploring case definitions for influenza-like illness. Infect Control Hosp Epidemiol 2015;36:241-8.

7. Minodier L, Charrel RN, Ceccaldi PE, et al. Prevalence of gastrointestinal symptoms in patients with influenza, clinical significance, and pathophysiology of human influenza viruses in faecal samples: what do we know? Virol J 2015;12:215.

8. Mertz D, Kim TH, Johnstone J, et al. Populations at risk for severe or complicated influenza illness: systematic review and meta-analysis. BMJ 2013;347:f5061.

9. Cromer D, van Hoek AJ, Jit M, et al. The burden of influenza in England by age and clinical risk group: a statistical analysis to inform vaccine policy. J Infect 2014;68:363-71.

10. Glezen WP, Greenberg SB, Atmar RL, et al. Impact of respiratory virus infections on persons with chronic underlying conditions. JAMA 2000;283:499-505.

11. Gill PJ, Ashdown HF, Wang K, et al. Identification of children at risk of influenza-related complications in primary and ambulatory care: a systematic review and meta-analysis. Lancet Respir Med 2015;3:139-49.

12. Loughlin J, Poulios N, Napalkov P, et al. A study of influenza and influenza-related complications among children in a large US health insurance plan database. Pharmacoeconomics 2003;21:273-83. 
13. Van Kerkhove MD, Vandemaele KA, Shinde V, et al. Risk factors for severe outcomes following 2009 influenza A (H1N1) infection: a global pooled analysis. PLoS Med 2011;8:e1001053.

14. Wong KK, Jain S, Blanton L, et al. Influenza-associated pediatric deaths in the United States, 2004-2012. Pediatrics 2013;132:796-804.

15. Mauskopf J, Klesse M, Lee S, et al. The burden of influenza complications in different high-risk groups: a targeted literature review. J Med Econ 2013;16:264-77.

16. Matias G, Taylor RJ, Haguinet F, et al. Modelling estimates of agespecific influenza-related hospitalisation and mortality in the United Kingdom. BMC Public Health 2016;16:481.

17. Peebles PJ, Dhara R, Brammer L, et al. Influenza-associated mortality among children - United States: 2007-2008. Influenza Other Respir Viruses 2011;5:25-31.

18. Myles PR, Semple MG, Lim WS, et al. Predictors of clinical outcome in a national hospitalised cohort across both waves of the influenza A/H1N1 pandemic 2009-2010 in the UK. Thorax 2012;67:709-17.

19. Prescott HC, Angus DC. Enhancing Recovery From Sepsis: A Review. JAMA 2018;319:62-75.

20. Hsu J, Santesso N, Mustafa R, et al. Antivirals for treatment of influenza: a systematic review and meta-analysis of observational studies. Ann Intern Med 2012;156:512-24.

21. Prevention and control of seasonal influenza with vaccines. Recommendations of the Advisory Committee on Immunization Practices-United States, 2013-2014. MMWR Recomm Rep 2013;62:1-43.

22. Grohskopf LA, Olsen SJ, Sokolow LZ, et al. Prevention and control of seasonal influenza with vaccines: recommendations of the Advisory Committee on Immunization Practices (ACIP) - United States, 201415 influenza season. MMWR Morb Mortal Wkly Rep 2014:63:691-7.

23. Centers for Disease Control ROCT. Influenza vaccination programe in Taiwan. 2018 https://www.cdc.gov.tw/File/Get/hsUDLK3zshcgPmz67rJVA (cited 2019 May 2).

24. McNeill A, Harris PR, Briggs P. Twitter Influence on UK Vaccination and Antiviral Uptake during the 2009 H1N1 Pandemic. Front Public Health 2016:4:26.

25. Chan TC, Hsiao CK, Lee CC, et al. The impact of matching vaccine strains and post-SARS public health efforts on reducing influenzaassociated mortality among the elderly. PLoS One 2010;5:e11317.

26. Center for Biomedical Resources of NHRI. Data Subsets. http://nhird. nhri.org.tw/en/Data Subsets.html (cited 16 Mar 2018).

27. Wu TS, Shih FY, Yen MY, et al. Establishing a nationwide emergency department-based syndromic surveillance system for better public health responses in Taiwan. BMC Public Health 2008;8:18.

28. Charlson M, Szatrowski TP, Peterson J, et al. Validation of a combined comorbidity index. J Clin Epidemiol 1994;47:1245-51.

29. Deyo RA, Cherkin DC, Ciol MA. Adapting a clinical comorbidity index for use with ICD-9-CM administrative databases. J Clin Epidemiol 1992;45:613-9.

30. Elixhauser A, Steiner C, Harris DR, et al. Comorbidity measures for use with administrative data. Med Care 1998;36:8-27.

31. Centers for Disease Control ROCT. Taiwan National Infectious Disease Statistics System. 2018 https://nidss.cdc.gov.tw/en/ CDCWNH01.aspx?dc=wnh (cited 15 Dec 2018).

32. Agresti A. An Introduction to Categorical Data Analysis. 2nd ed. Hoboken, New Jersey: Wiley-Interscience, 2007.

33. Hosmer DW, Lemeshow S. Applied Logistic Regression. 2nd ed. Hoboken: New Jersey: John Wiley \& Sons, 2000.

34. World Health Organization. Full report for the SAGE meeting. 2017 http://www.who.int/immunization/policy/sage/en/ (cited 2018 Mar 16).

35. Centers for Disease Control and Prevention. Prevention and Control of Seasonal Influenza with Vaccines, 2017-18. $2017 \mathrm{https}$ ://www.cdc. gov/flu/professionals/acip/index.htm (cited 16 Mar 2018).

36. Public Health England. Flu Plan Winter 2017/18. 2017 https://www. gov.uk/government/uploads/system/uploads/attachment_data/file/ 600532/annual_flu_plan_2017to2018.pdf (cited 16 Mar 2018).

37. Villalón-Letelier F, Brooks AG Saunders PM, et al. Host Cell Restriction Factors that Limit Influenza A Infection. Viruses 2017;9:376

38. Estabragh ZR, Mamas MA. The cardiovascular manifestations of influenza: a systematic review. Int J Cardiol 2013;167:2397-403.

39. Smeeth L, Thomas SL, Hall AJ, et al. Risk of myocardial infarction and stroke after acute infection or vaccination. N Engl J Med 2004;351:2611-8.

40. Chertow DS, Memoli MJ. Bacterial coinfection in influenza: a grand rounds review. JAMA 2013;309:275-82.

41. Sheth AN, Althoff KN, Brooks JT. Influenza susceptibility, severity, and shedding in HIV-infected adults: a review of the literature. Clin Infect Dis 2011;52:219-27.
42. Kunisaki KM, Janoff EN. Influenza in immunosuppressed populations: a review of infection frequency, morbidity, mortality, and vaccine responses. Lancet Infect Dis 2009;9:493-504.

43. Noymer A. The 1918 influenza pandemic hastened the decline of tuberculosis in the United States: an age, period, cohort analysis. Vaccine 2011;29 Suppl 2(29 Suppl 2):B38-B41.

44. Walaza S, Tempia S, Dawood $\mathrm{H}$, et al. Influenza virus infection is associated with increased risk of death amongst patients hospitalized with confirmed pulmonary tuberculosis in South Africa, 2010-2011. BMC Infect Dis 2015;15:26

45. Tecklenborg J, Clayton D, Siebert S, et al. The role of the immune system in kidney disease. Clin Exp Immunol 2018;192:142-50.

46. Allard R, Leclerc P, Tremblay C, et al. Diabetes and the severity of pandemic influenza A (H1N1) infection. Diabetes Care 2010;33:1491-3.

47. Shang M, Blanton L, Brammer L, et al. Influenza-Associated Pediatric Deaths in the United States, 2010-2016. Pediatrics 2018:141:e20172918.

48. Edwards KM. What Have We Learned About Influenza Deaths in Children and How Can We Do Better? Pediatrics 2018:141:e20174313.

49. López-Campos JL, Tan W, Soriano JB. Global burden of COPD. Respirology 2016;21:14-23.

50. Ding B, Small M, Bergström G, et al. COPD symptom burden: impact on health care resource utilization, and work and activity impairment. Int J Chron Obstruct Pulmon Dis 2017:12:677-89.

51. Cheng SL, Chan MC, Wang CC, et al. COPD in Taiwan: a National Epidemiology Survey. Int J Chron Obstruct Pulmon Dis 2015;10:2459-67.

52. Ma YC, Lin CC, Yang SY, et al. Time Trend Analysis of the Prevalence and Incidence of Diagnosed Asthma and Traditional Chinese Medicine Use among Adults in Taiwan from 2000 to 2011: A Population-Based Study. PLoS One 2015;10:e0140318.

53. Centers for Disease Control ROCT. Guideline for Influenza Antivira Medications. 2018 https://www.cdc.gov.tw/File/Get/5ILQw9Gsd8Mm D6A9TWJj3Q (cited 2 May 2019).

54. Lee MG, Lee CC, Lai CC, et al. Preadmission statin use improves the outcome of less severe sepsis patients - a populationbased propensity score matched cohort study. Br J Anaesth 2017;119:645-54.

55. Obermeyer Z, Lee TH. Lost in Thought - The Limits of the Human Mind and the Future of Medicine. N Engl J Med 2017;377:1209-11.

56. Yang L, Chan KP, Lee RS, et al. Obesity and influenza associated mortality: evidence from an elderly cohort in Hong Kong. Prev Med 2013;56:118-23.

57. Karki S, Muscatello DJ, Banks E, et al. Association between body mass index and laboratory-confirmed influenza in middle aged and older adults: a prospective cohort study. Int $J$ Obes 2018;42:1480-8.

58. Sun $Y$, Wang $Q$, Yang $G$, et al. Weight and prognosis for influenza $A(H 1 N 1) p d m 09$ infection during the pandemic period between 2009 and 2011: a systematic review of observational studies with metaanalysis. Infect Dis 2016;48:813-22.

59. van Zyl-Smit RN, Brunet L, Pai M, et al. The convergence of the global smoking, COPD, tuberculosis, HIV, and respiratory infection epidemics. Infect Dis Clin North Am 2010;24:693-703.

60. Tamerius J, Nelson MI, Zhou SZ, et al. Global influenza seasonality: reconciling patterns across temperate and tropical regions. Environ Health Perspect 2011;119:439-45.

61. Feng C, Li J, Sun W, et al. Impact of ambient fine particulate matter (PM2.5) exposure on the risk of influenza-like-illness: a time-series analysis in Beijing, China. Environ Health 2016;15:17.

62. Fiore AE, Uyeki TM, Broder K, et al. Prevention and control of influenza with vaccines: recommendations of the Advisory Committee on Immunization Practices (ACIP), 2010. MMWR Recomm Rep 2010;59:1-62.

63. Brundage JF. Interactions between influenza and bacterial respiratory pathogens: implications for pandemic preparedness. Lancet Infect Dis 2006;6:303-12.

64. Jacobs JH, Viboud C, Tchetgen ET, et al. The association of meningococcal disease with influenza in the United States, 19892009. PLoS One 2014:9:e107486.

65. Lee N, Choi KW, Chan PK, et al. Outcomes of adults hospitalised with severe influenza. Thorax 2010;65:510-5

66. Cowling BJ, Chui CSL, Lim WW, et al. Use of influenza antivirals in patients hospitalized in Hong Kong, 2000-2015. PLoS One 2018;13:e0190306

67. Domínguez A, Romero-Tamarit A, Soldevila N, et al. Effectiveness of antiviral treatment in preventing death in severe hospitalised influenza cases over six seasons. Epidemiol Infect 2018;146:799-808. 